\title{
Medley of Spirals from Cyclic Cellular Automata
}

\author{
Clifford A. Reiter
}

Department of Mathematics, Lafayette College, Easton, PA 18042-1781, USA

(preprint)

\begin{abstract}
Cyclic cellular automata on the integer planar lattice are known to typically evolve through distinct phases ending with minimal periodic terminal states that usually appear as intertwined spirals. Here we explore the diversity of spirals that arise from nonstandard neighborhoods on the integer lattice and from looking at the automata on quasi-crystalline arrangements of cells. We see that phase transitions and development of spirals are almost ubiquitous yet the particular form of the spirals is very dependent upon the particulars of the underlying neighborhoods; in fact the spiral forms echo the neighborhoods. The quasi-crystalline illustrations provide much more subtle echoes in the spiral forms that show artifacts from the non-periodic local symmetry that occurs.
\end{abstract}

Keywords: Automata, self organization, phase transition, quasi-crystals, cyclic state automata

\section{Introduction}

A cellular automaton is a collection of cells that in each time step are in a "state"; usually the set of allowed states for each cell is finite. The cells are affected by a specific local neighborhood and a local rule such that the cells evolve from one time step to the next according to the rule. Cellular automata are interesting because of their simplicity and complex behavior [1-3]. The most famous automaton is Conway's Game of Life which was popularized by Martin Gardner's Scientific American columns [4-5]. That automaton runs on the integer lattice with nearest eight neighbors and two possible states that are updated according to a simple rule. It is intriguing because periodic structures occur, as do moving configurations and generators. In fact, it is known to be capable of universal computation [6].

Cyclic cellular automata were introduced by David Griffeath [7] and described by Dewdney [8] in Scientific American in 1989 where they were called cyclic state automata. We will refer to them as cyclic cellular automata (CCA). When CCA are applied to a random initial configuration, they typically evolve through distinct phases that have different appearances. The end result is visually dramatic, periodic spirals that self-organize. Spiral formation arises in physical situations [2-3, 9-10] and in other models [1-3, 7, 10-13]. While not every question about these automata can be answered, it is possible to see why organized structures should develop, as explained in [7-8, 10], and will be described below. Being able to explain this rich behavior is an unusual and wonderful feature of these automata. Generalizations of CCA to wider neighborhoods and thresholds has been studied as well [7, 10, 12].

A cyclic cellular automaton is defined as an automaton where each cell takes one of $N$ states $0,1,2, \ldots, N-1$ and a cell in state $i$ changes to state $i+1 \bmod N$ at the next time step if it has a neighbor that is in state $i+1 \bmod N$, otherwise it remains in state $i$ at the next time step. The most classic CCA are applied on the 2-dimensional integer lattice with von Neuman neighborhoods (nearest four NWES neighbors). However, this rule can be applied to any configuration of cells and any definition of neighborhood in any dimension. In fact, it can be applied to any graph. 
In this investigation we explore CCA on a rich variety of planar graphs: these include several types of nearest-neighbor neighborhoods on the integer lattice and quasi-crystalline graphs. There are many kinds of neighborhoods can be formed even in the case where only nearest neighbors are considered and the resulting spirals include classical diamonds, squares, and variants that echo the neighborhood in an inverted sense. The quasi-crystalline arrangements of cells lead to many sided spirals that are echoes of more subtle local symmetry appearing in those configurations.

\section{Debris, Defects and Demons}

Before giving definitions, we give a classic illustration. Figure 1 shows CCA using von Neuman neighborhoods on a 500 by 500 array of cells. Periodic boundary conditions are used so that cells on the right edge have neighbors from the left edge and analogous comments apply to the left, top and bottom edges. Four different time steps are shown in Figure 1. The automata were implemented in $\mathrm{J}$ [14] using the automata templates from [15]. The cyclic cellular automata uses $N=14$ states and the initial states for each cell are chosen randomly and uniformly from those states. The states are shown cyclically with hue, running from red through intermediates to green and then blue and magenta as the states run from 0 to $N-1$. The upper left portion of the figure occurs after 75 time steps and one can observe rough "debris” regions that have not substantially evolved and "droplets" of color, with some waves of changes moving across them. The upper right shows the result after 150 time steps and we see the droplets cover a majority of the region, but some spiral "defects" have begun to evolve. After 225 time steps, shown on the lower left, we see many spirals have formed and some of them have grown quite large. Here the shortest possible nontrivial periodicity that can occur is 14 and the black pixels mark the edges of regions that have repeated with that optimal periodicity. The spirals (defects) that are surrounded by black pixels have optimal period, and are called "demons". The bottom right of the figure shows the situation after 975 time steps. At this point the demons have overtaken most of the other spirals and the black pixels surround small regions that are defects with periods higher than 16, which will soon also be overtaken by the demons. Many movies showing the evolution of the CCA described in this paper may be found at [16]. Watching the phase transitions evolve reinforces the dynamic nature of these processes.

Following [7-8] we describe these phases more carefully. The bond between two neighbors is open if the difference between their states is $\{-1,0,1\} \bmod N$. Otherwise the bond is closed. Note that once a bond is open it must remain open for all future time. A site is considered debris at a given time if it has no open bonds with its neighbors. The connected components of the non-debris sites are called droplets. A loop within a droplet using open bonds could possibly include only bonds $\{-1,0,1\} \bmod N$. If one takes a running sum (not $\bmod N$ ) of the differences $\bmod N$ (so each difference is from $\{-1,0,1\}$ ) along such a loop, then the total must be zero mod $N$ since each cell value appears once in a positive and negative sense. Thus, the sum of the differences must be a multiple of $N$. If the multiple is not zero, then the loop is part of a defect. That property of the loop will be preserved as the automaton evolves, and each cell in a defect must eventually continue cycling through the states $0,1,2, \ldots, N-1,0, \ldots$ forevermore; although it may take more than one time step between each change of state. Eventually every cell neighboring such a loop must cycle. Then their neighbors must cycle. Eventually every cell in such a droplet must cycle, and thus the droplet must grow since every neighbor of the droplet will eventually join the droplet since the neighbor in the droplet will eventually cycle through all values. Of course the speed of such cycling need not be constant until it is part of a demon. If a 
defect loop runs through all states as efficiently as possible on the lattice (minimal period having no unnecessary 0 bonds) then it is a demon. Given random initial states for all the cells on an infinite lattice, demons are expected to occur somewhere with probability one. Thus, demon domination is the expected long term state.

Figure 2 shows zooms into two of the spirals from the end state of the experiment shown in Figure 1. The closed bonds are demarcated by black lines. On the left is a demon since if one marches around the "L" shaped closed bonds, one moves through the 14 states using 14 cells crossing 14 bonds; all those bonds correspond to one level change in state, all the same in a positive or negative sense. That property persists for all future times and thus that spiral is a demon. The spiral on the right is a defect, but not a demon. Any short path around the closed bonds using left-right and up-down bonds requires more than 14 cells and visits yellow and violet cells twice. Such a loop must persist but changes can (and will, with probability one) occur to the closed bonds so that this region becomes part of a more efficient, minimal period, defect that is a demon.

However, the arrangement of cells may not allow for a demon loop of length $N$. For example, with von Neuman neighborhoods, it is not possible for a loop to return to itself using an odd number of bonds. Thus, if $N=15$ then a demon loop must contain at least one bond where there is no change in state. Figure 3 shows this situation where a loop around the closed bonds visits light green twice. Also note debris, droplets, defects and demons are all apparent after 255 time steps.

\section{Moore neighborhoods}

We now turn to discussing other neighborhood patterns using sub-neighborhoods of the 3 by 3 neighborhood surrounding the cell. We number a 3 by 3 neighborhood so that the center has number 4 as shown in Figure 4. Letting $P$ denote the neighborhood pattern, we write $P=1357$ for the von Neuman neighborhoods used in the previous section. The complete Moore neighborhood thus has pattern given by $P=01235678$. The behavior of the CCA with this sense of neighbor is for the most part qualitatively similar that what we saw in Figure 1, but some distinctions should be made. Figure 5 shows the $N=20$ state evolution at times 63, 105, 147, and 231, respectively. Notice the droplet formation, spirals developing, dominating and becoming ubiquitous. However, unlike the $P=1357$ pattern, the square spirals tend to have their edges parallel to the lattice.

However, the situation is different when the number of states is different. Notice that on the upper left of Figure 6, which corresponds to $N=8$, the cells settle into a periodic (period 8) pattern that involves grainy patches, but it would be an exaggeration to suggest these are spirals. The upper right shows the final periodic state when $N=16$ and the lower left shows it for $N=26$. Note that the spirals are much larger when the number of states is larger. We see very typical spiral formation in those cases. The lower right show $N=40$ and here the evolution stops after 96 steps. At this point the number of states has gotten so large that many cells and small droplets are surrounded by closed bonds and no evolution continues. Note that this is a finite scale effect in the sense that if the array of cells was infinite, with probability one we would expect there to be a defect loop somewhere and that would force every cell into a periodic state in finite time. 


\section{Restricted Neighborhoods}

Next we consider the neighborhood $P=0123567$ with $N=20$, shown in the upper left of Figure 7 at a time when the demons are a sound majority. In this neighborhood pattern there are no connections running to a cell from the southeast, and hence a spiral wave can not move northwest. The result is the truncated spiral shown. On the upper right of Figure 7 we see the spirals arising from $P=012357$ and $N=18$. This time the spirals are truncated on the top left and right. The lower left shows $P=01357, N=16$ spirals. It is no surprise that if we remove the last corner and go to $P=1357$ we get the diamond spirals seen in Figure 1. Lastly, the lower right portion of Figure 7 shows $P=0157$ with $N=13$ and we see rather asymmetric spirals form. In each case, we see the spiral form is an inverted echo of the neighborhood.

In Figure 8 we consider some more extreme patterns. The upper left shows $P=0268$ with $N=10$. Note that here cells are only connected diagonally so that alternate squares in a checkerboard fashion create two independent arrangements like the von Neumann neighborhoods rotated 45 degrees. The upper right shows the pattern $P=027$ when $N=10$. It is quite intriguing to have triangular spirals on a square lattice. For purposes of obtaining defects, edges are only "one-way” and loops using those directed edges must have a multiple of four in length. In particular, the demons seen here have period 12. Movies showing the development of these $P=027$ CCA are slightly different due to the few edges [16]. The lower left shows $P=135$ with $N=9$ where it is not possible for spirals to form since there is no way for a cell to increase in state from below. However, given the periodicity of this finite version, droplets form and optimal waves develop. That portion of the figure show a state when droplets dominant and as the dominating waves begin to develop. In the lower right portion of the figure we have $P=015$ with $N=8$; again no spirals, but optimal waves form on these finite periodic domains.

\section{Quasi-crystal neighborhoods}

We now turn to neighborhoods that have been created by canonical projection. Typically these result in graphs with quasi-crystalline structure. These have local patterns, but not global symmetry. In special cases ordinary crystalline lattices can be formed and it is also possible for very carefully chosen quasi-crystalline arrangements to have a rotational symmetry. Many examples of quasi-crystalline patterns arising from canonical projection are shown in [17] where a description of the creation process may also be found. Figure 9 shows a quasi-crystalline arrangement generated from 5-d that results in patterns with local 5-fold symmetry. While there are not any periodicities, any patch that occurs will occur repeatedly in infinite versions of these arrangements; this is a special arrangement with a 5-fold rotational symmetry, but due to the random nature of the initial conditions, that is of no consequence.

For the CCA on such an arrangement, we take the cells to correspond to vertices and edges show which cells are neighbors. We created such an arrangement with slightly over 600k vertices and render CCA images so that the state of each cell typically shown by the color of one or two nearest pixels. The implementation uses clipped boundary conditions in the sense that edge cells simply have fewer neighbors than typical interior cells. For example, unlike periodic boundary conditions, cells on the right edge are not connected to cells left edge. Figure 10 shows the development of the CCA on that arrangement with spiral formation on the left after 78 time steps and on the right after 208 time steps. The CCA evolves through the expected droplets to defect spirals with demons coming to dominate. Note that on this arrangement of cells, it is not possible to have loops using an odd number of edges. Thus, when there is an odd number of 
states, $N$, the demons will have periodicity $N+1$. Notice that the spirals are much rounder than those seen previously, but that there is a 10-sided appearance on close inspection.

Figure 11 shows two small patches of the crystalline neighborhoods obtained from canonical projection from three and six dimensions. On the left portion of the figure, each cell has six neighbors, so this is the classical hexagonal lattice. On the right portion, some cells have six neighbors while others have three, so this is a restricted hexagonal lattice. Larger versions of these patterns are used to create the CCA illustrated in Figure 12. Here we show the development at a time when droplets are still apparent, defects cover most of the image and demons (interior of the black marked regions) have begun to develop. In both cases there are $N=14$ states. On the left, $t=90$, while on the right, $t=165$. Given the fact that there are fewer edges in the version on the right, it is reasonable to expect the spirals to take longer to organize. Projection from 4-d also gives a crystalline lattice; the same one used to create Figure 1.

Figure 13 shows a portion of the quasi-crystalline arrangement of cells that arises from canonical projection from 7-d and 8-d. The versions we use for the CCA use over 708k and 126k cells respectively. We again consider $N=14$ states. Figure 14 shows these CCA after 855 and 645 steps respectively. At these times the demons are highly developed. The 14 -sided spirals on the left side of the figure have a very smooth appearance. The 8-sided spirals seen on the right side of the figure are somewhat fuzzy due to the fact that several pixels correspond to each cell.

We have seen that the CCA using a close sense of neighbor via patterned subneighborhoods of 3 by 3 patches or via arrangements arising from canonical projection typically evolve through the droplet, defect and demon phases. The spirals that self-organize are very sensitive to the particular neighborhood arrangement and tend to echo the local connection configurations.

\section{References}

[1] Ilachinski A. Cellular Automata: a Discrete Universe. Singapore: World Scientific; 2001. [2] Schiff JL. Cellular automata: a discrete view of the world. Hoboken: John Wiley \& Sons, Inc; 2008.

[3] Wolfram S. A new kind of science, Champaign: Wolfram Media; 2002.

[4] Gardner M. The fantastic combinations of John Conway’s new solitaire game of "life”, Scientific American 1970;223(4):120-123.

[5] Gardner M. On cellular automata, self-replication, the Garden of Eden and the game "life", Scientific American 1971;224(4):112-117.

[6] Weisstein EW. Universal Cellular Automaton. From MathWorld: A Wolfram Web Resource. http://mathworld.wolfram.com/UniversalCellularAutomaton.html.

[7] Fisch R, Gravner J, Griffeath D. Cyclic cellular automata in two dimensions. In Spatial

Stochastic Processes, Alexander KS, Wadkins JC, ed. Boston: Birkhäuser 1991; 171-185.

[8] Dewdney AK. Computer recreations: a cellular universe of debris, defects and demons.

Scientific American 1989;261(2):102-105.

[9] Bjornstad ON, Bacompte J. Synchrony and second order spatial correlation in host-parasitoid hierarchies. Journal of Animal Ecology 2001;70:924-933.

[10] Durrett R, Griffeath D. Asymptotic Behavior of Excitable Cellular Automata. Journal of Experimental Mathematics 1993;2(3):183-208.

[11] Dong CY, Long JT, Reiter CA, Staten C, Umbrasas R. A cellular model for spatial population dynamics. Computers and Graphics: to appear.

[12] Fisch R, Gravner J, Griffeath D. Threshold-range scaling of excitable cellular automata. 
Statistics and Computing 1991;1:23-99.

[13] Griffeath D, Moore, C (editors). New Constructions in Cellular Automata. New York: Oxford University; 2003.

[14] Jsoftware 6.02, http://www.jsoftware.com.

[15] Reiter CA. Fractals, visualization, and J. 3rd ed. Lulu; 2007.

[16] Reiter CA. Medley of spirals from cyclic cellular automata auxiliary materials. http://www.lafayette.edu/ reiterc/mvq/mscca/index.html; 2009.

[17] Reiter CA. Atlas of quasicrystalline tilings. Chaos, Solitons \& Fractals 2002;14:937-963 and http://www.lafayette.edu/ reiterc/aqct/index.html.

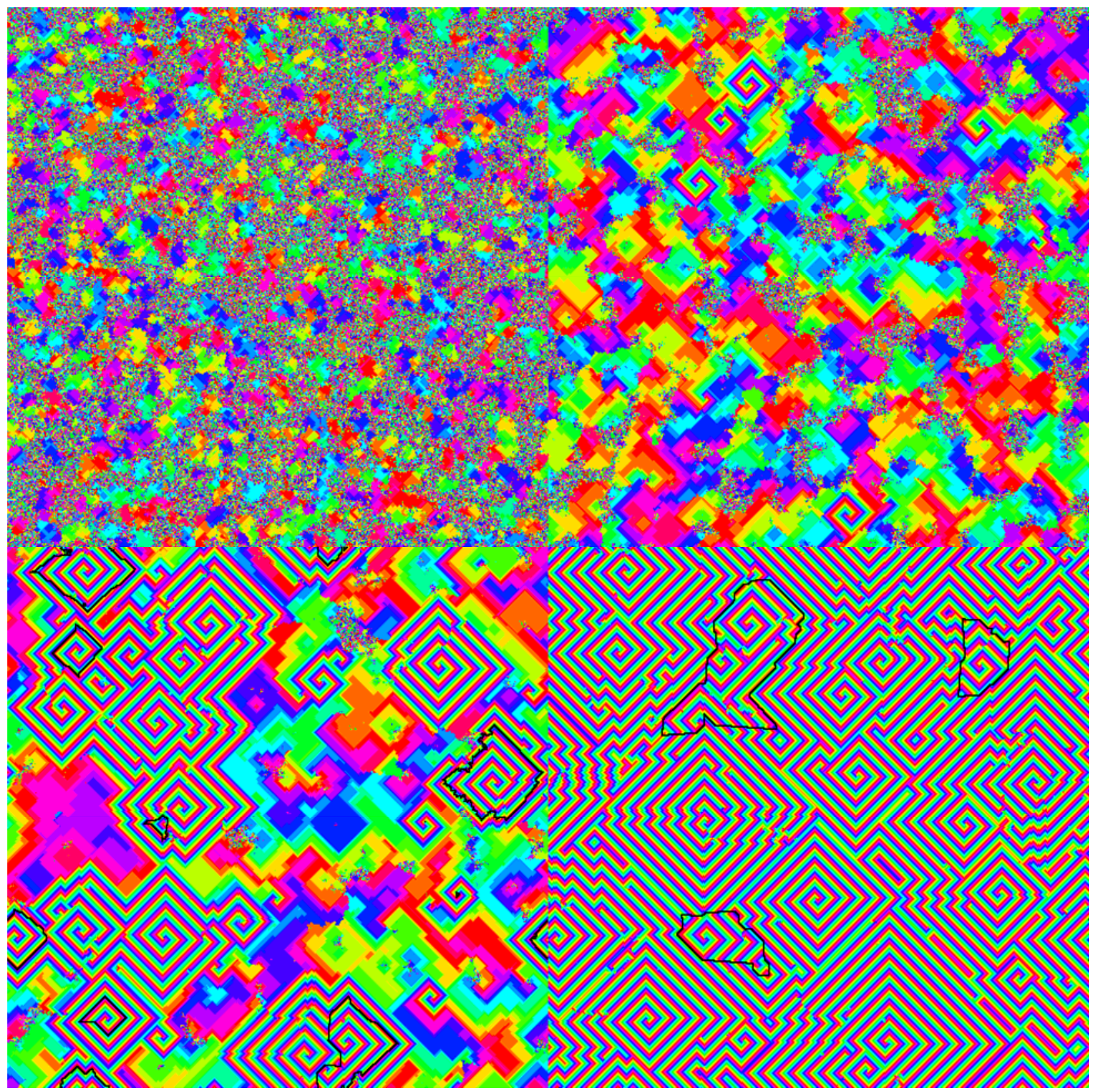

Figure 1. Classic CCA with $N=14$ showing droplet formation, spiral formation and demon domination. 


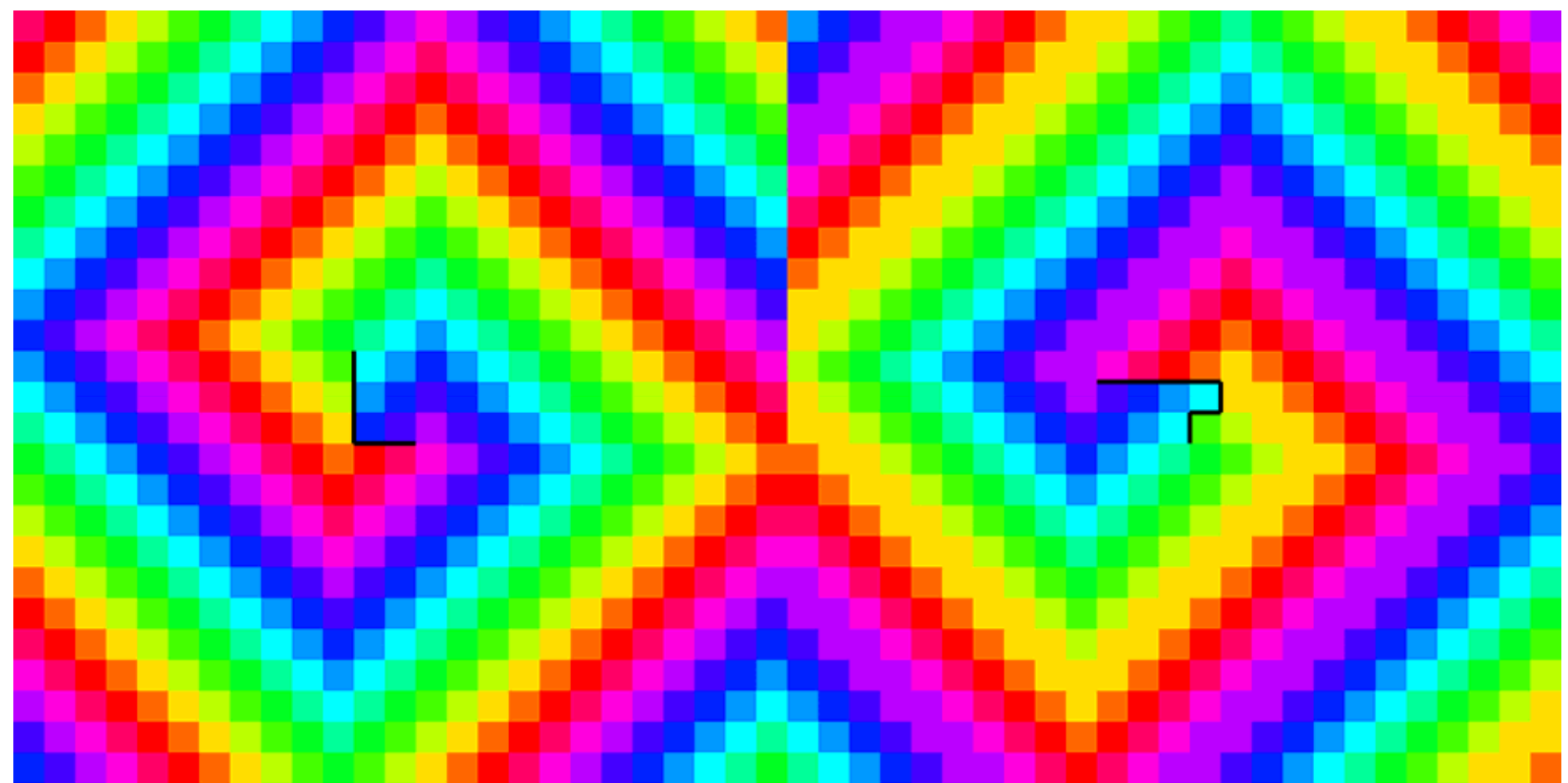

Figure 2. CCA with $N=14$. A demon and a non-demon defect with inactive bonds shown in black.

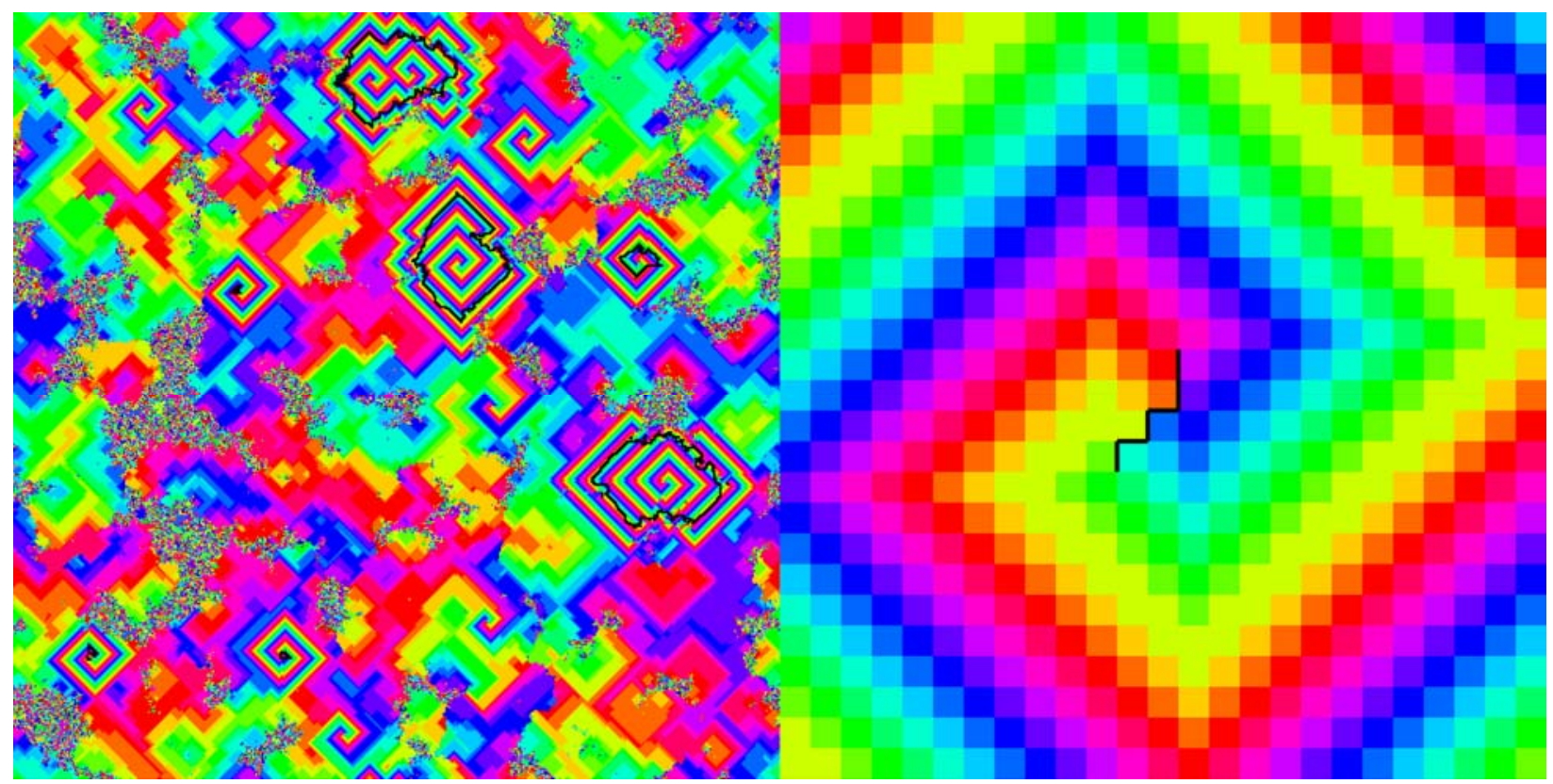

Figure 3. Demons forming and a demon loop at $t=255$ with $N=15$; this demon needs 16 bonds. 


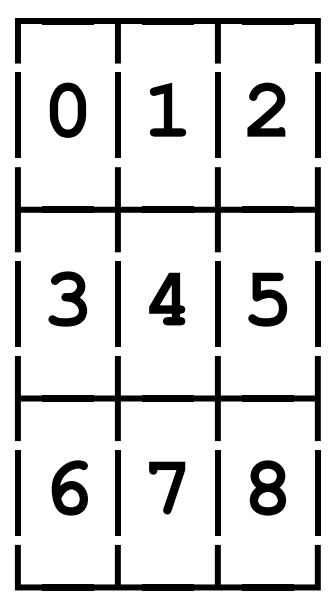

Figure 4. Center cell 4 with numbered neighbors. 


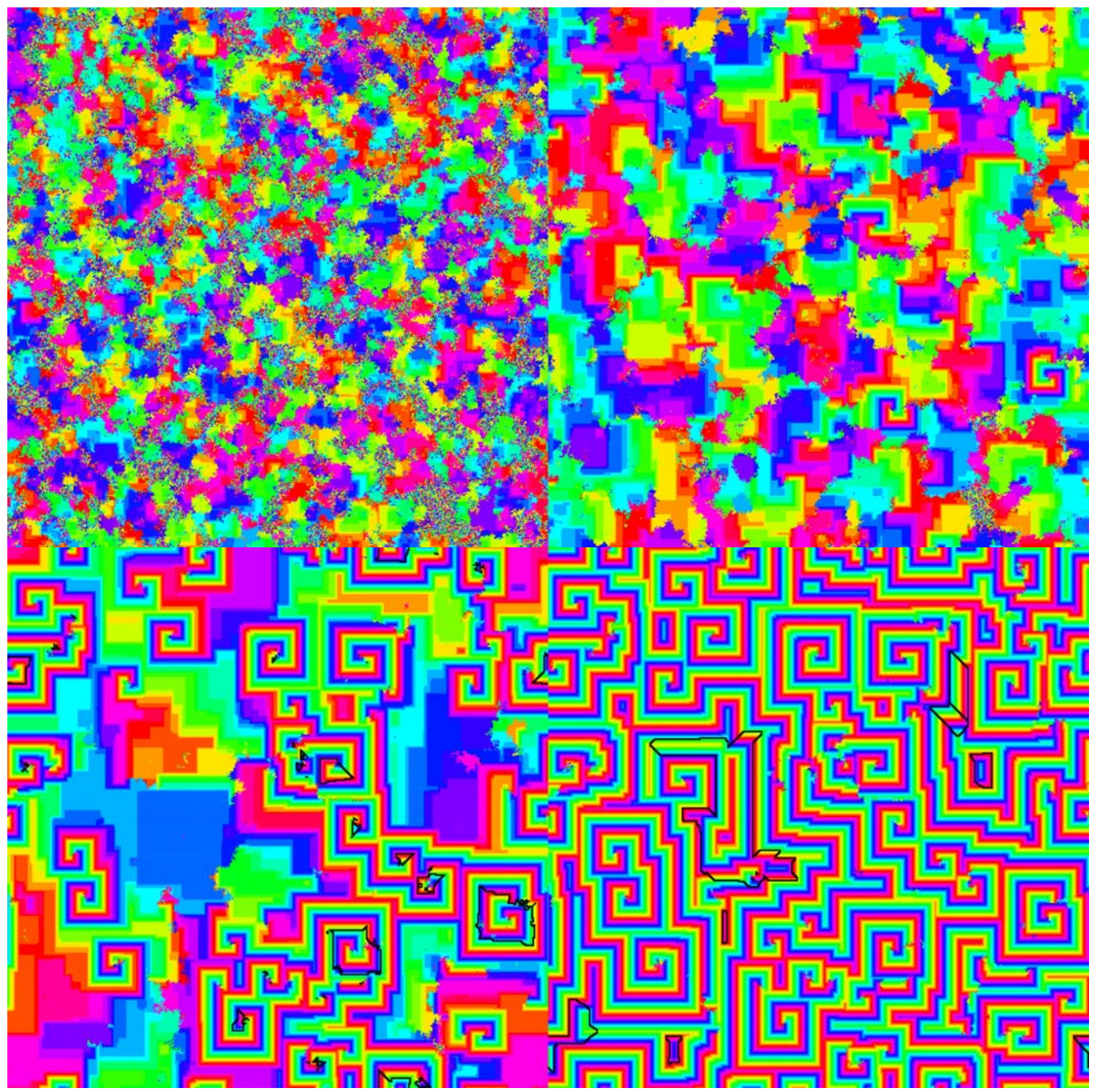

Figure 5. Using $N=20$ states and pattern $P=01235678$ at times $t=63,105,147,231$. 


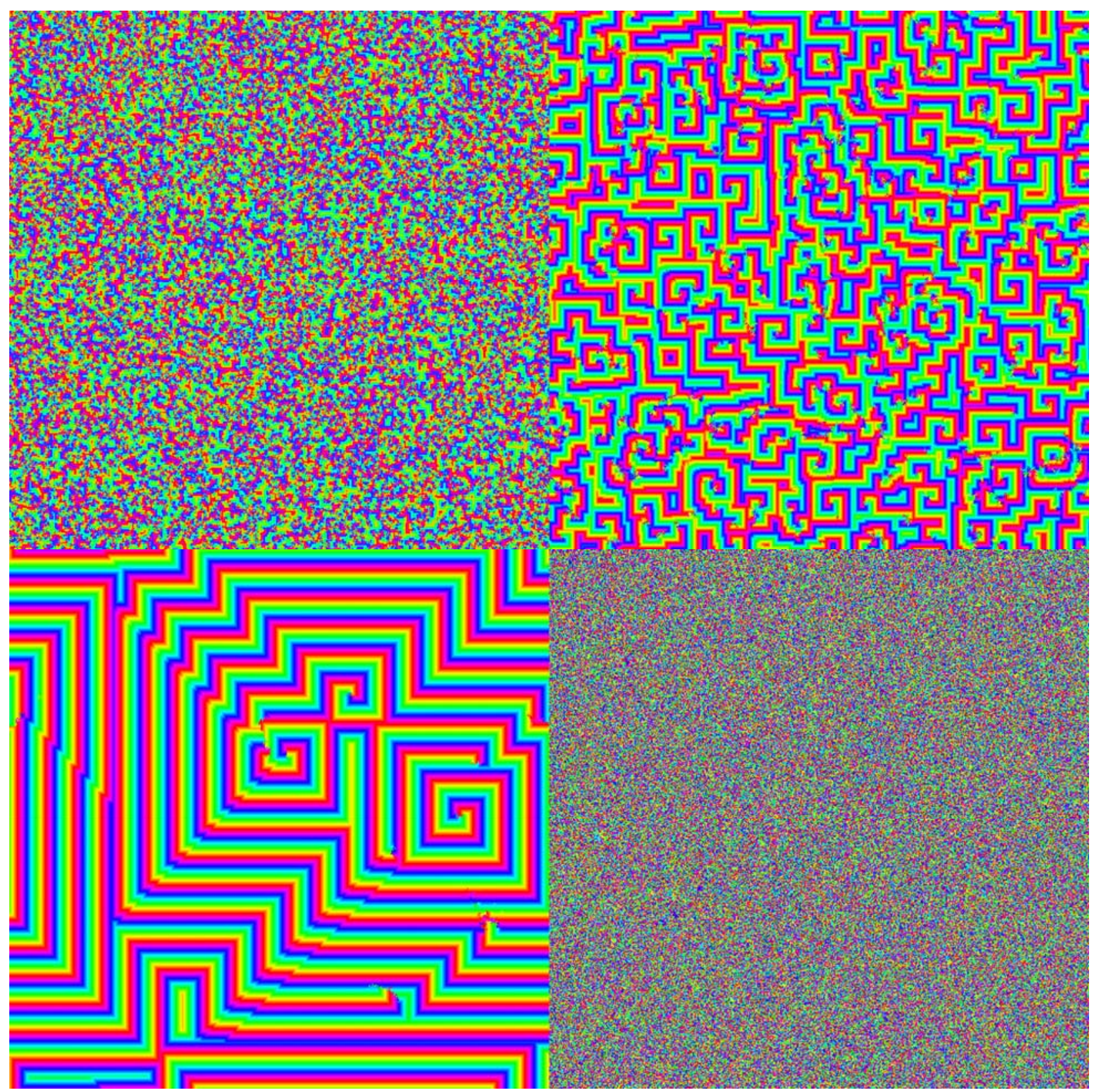

Figure 6. Using $N=8,16,26,40$ states and pattern $P=01235678$ at final periodic times. 


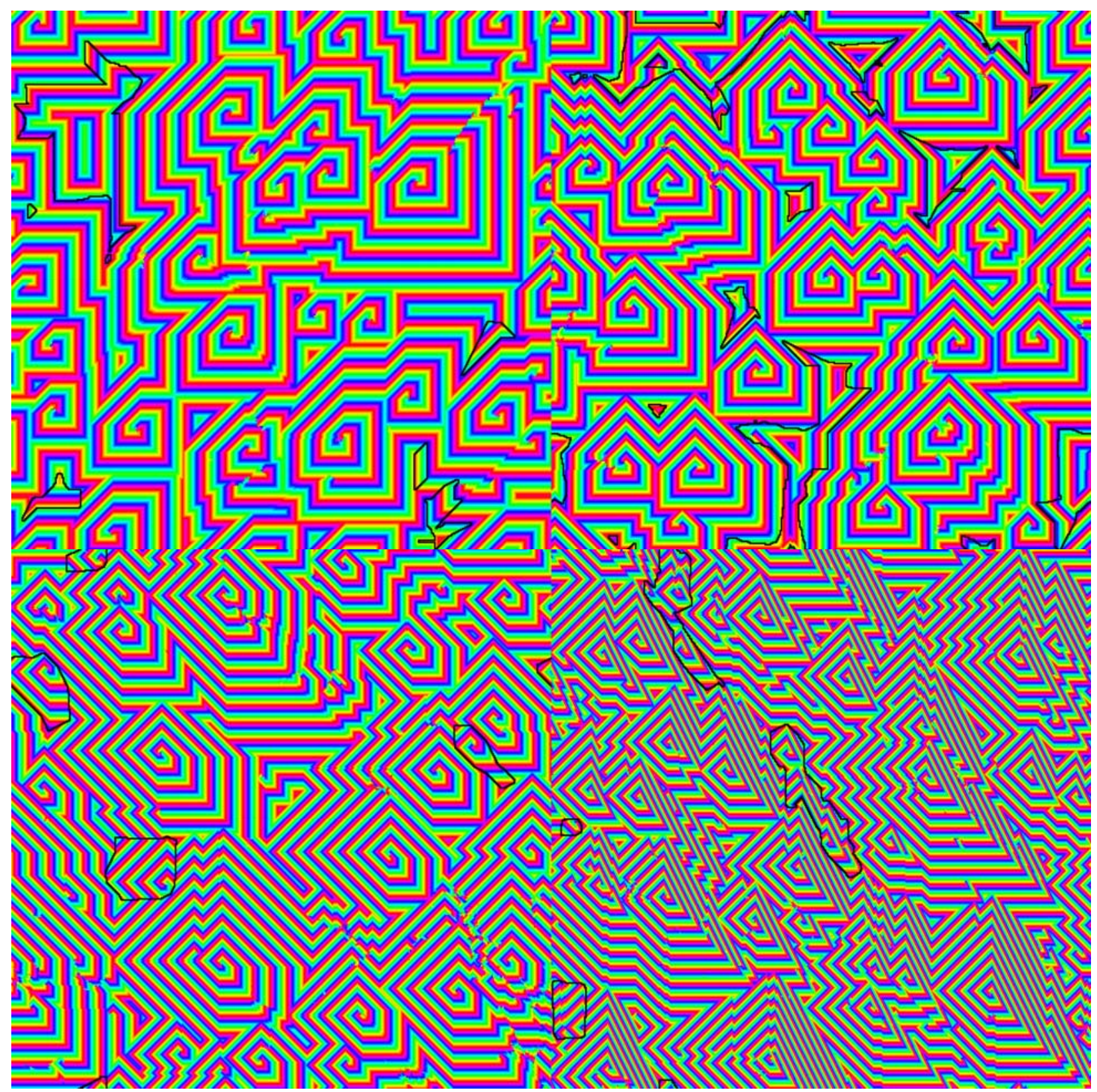

Figure 7. Neighborhoods using patterns $P=0123567, P=012357 P=01357$ and $P=0157$. 


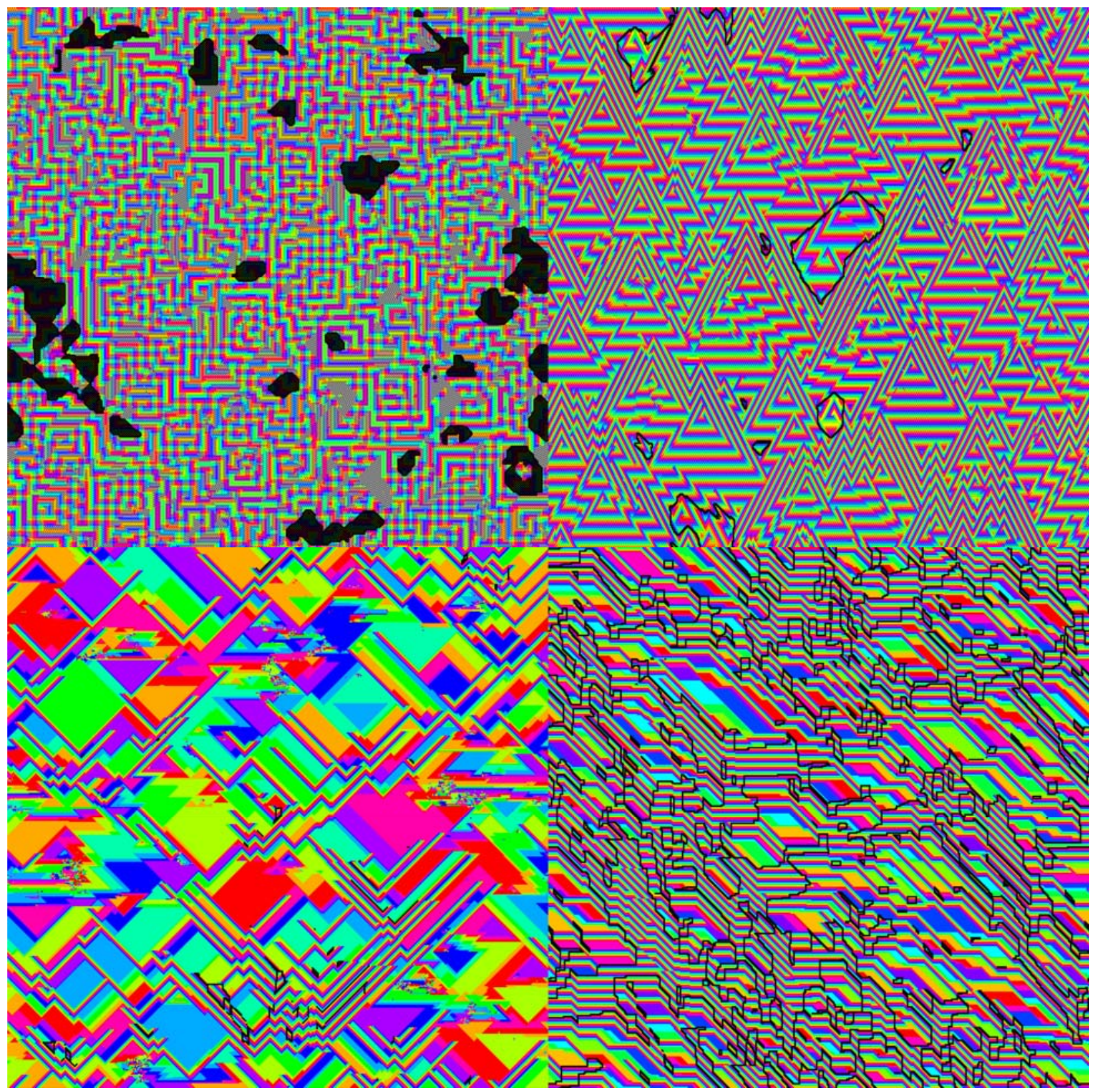

Figure 8. Extreme patterns $P=0268, P=027, P=135$, and $P=015$. 




Figure 9. A quasi-crystal created by canonical projection from 5-dimensions. 


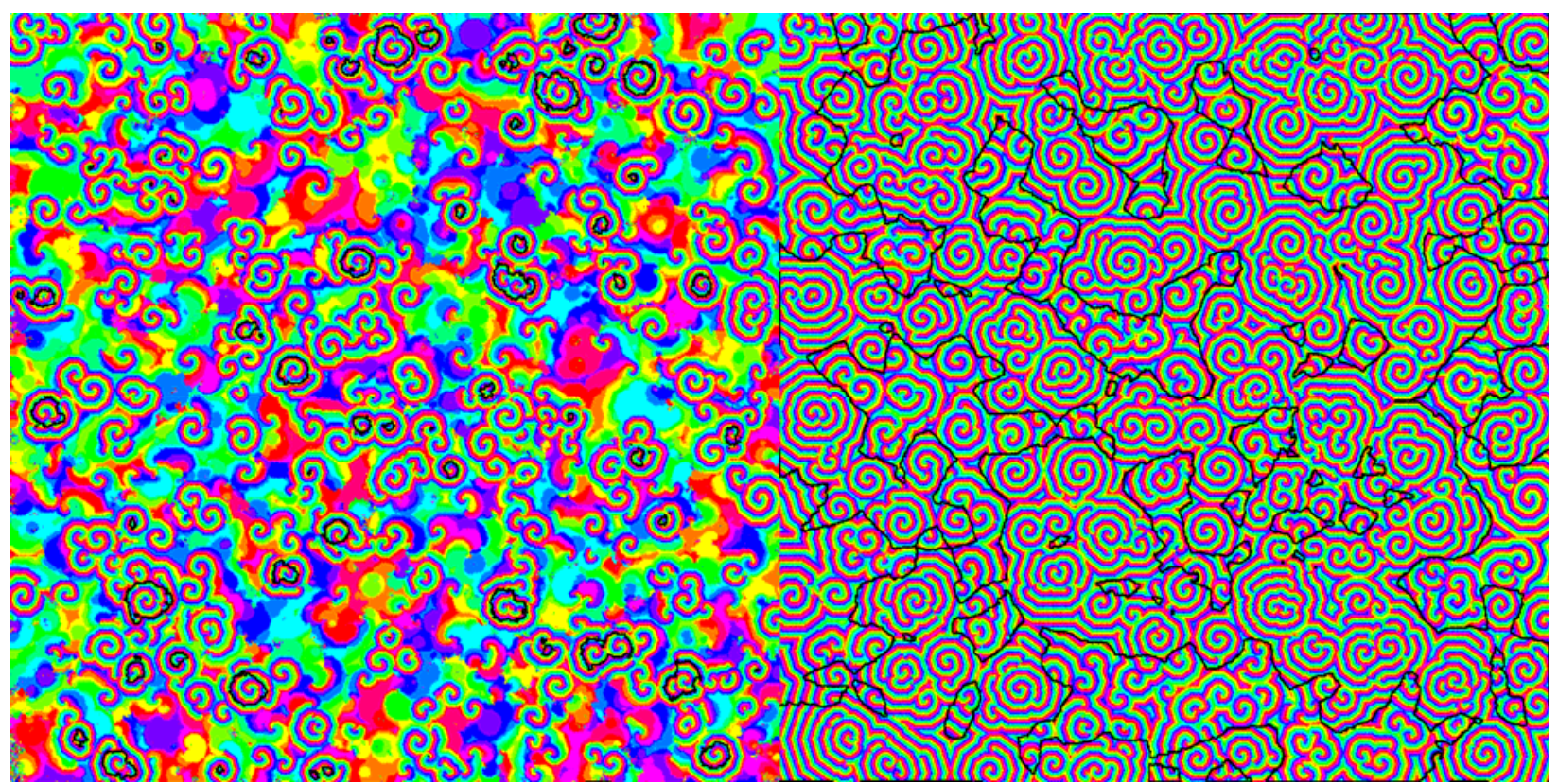

Figure 10. Spiral formation on a quasi-crystal with 5-fold local symmetry. 


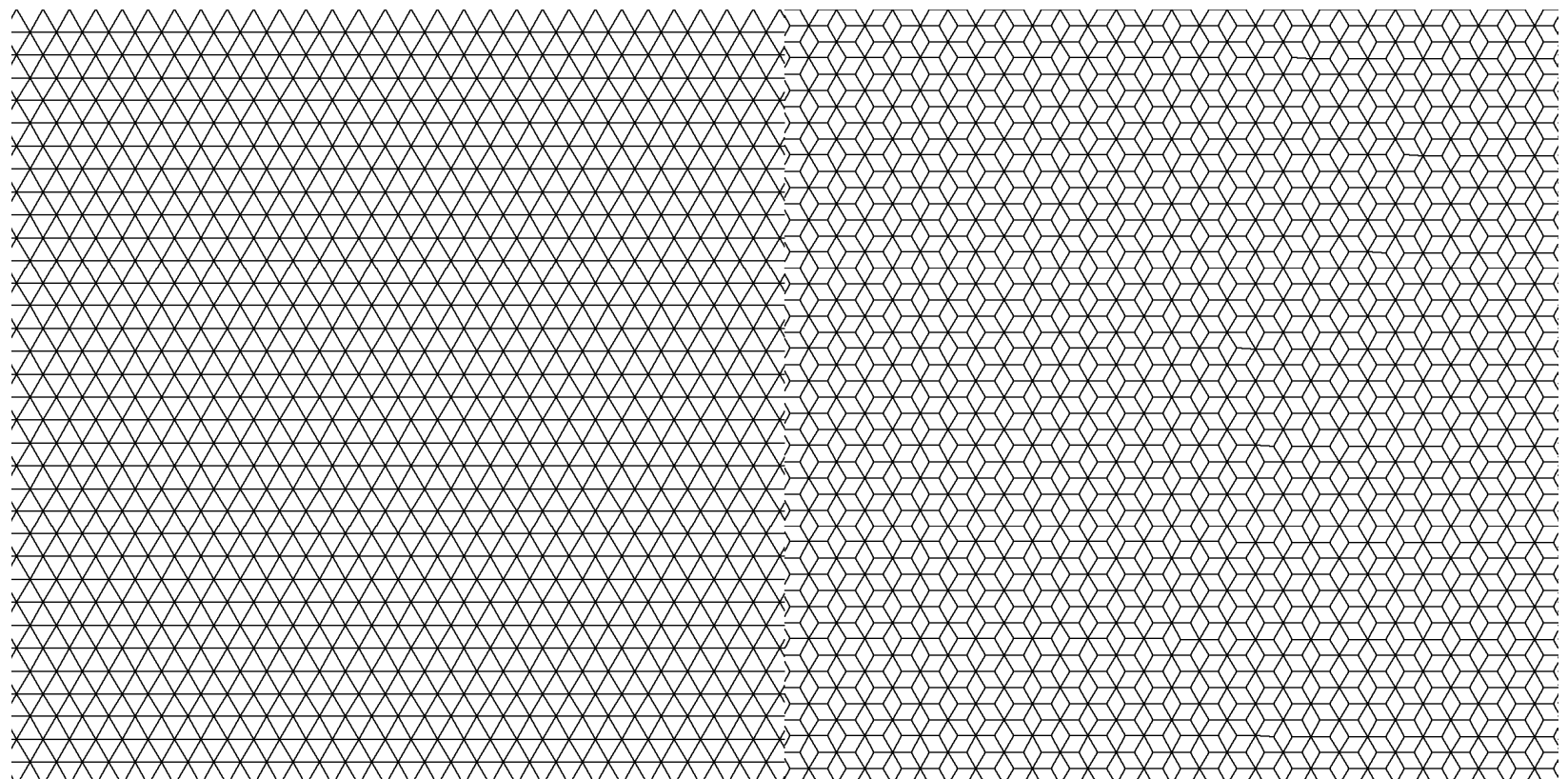

Figure 11. Crystalline lattices arising from canonical projection from 3-d and 6-d.

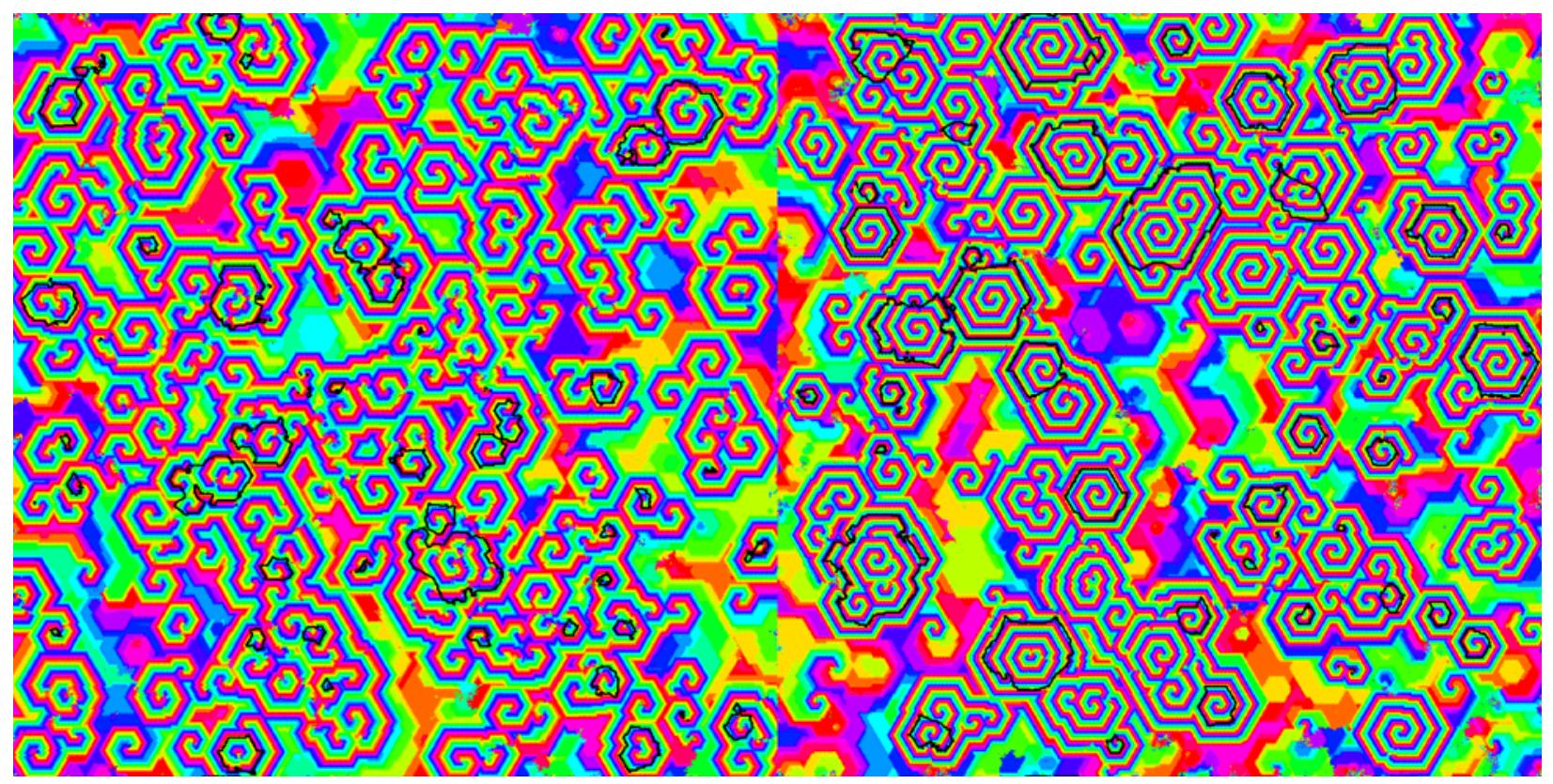

Figure 12. CCA with $N=14$ on crystalline hexagonal lattices. 


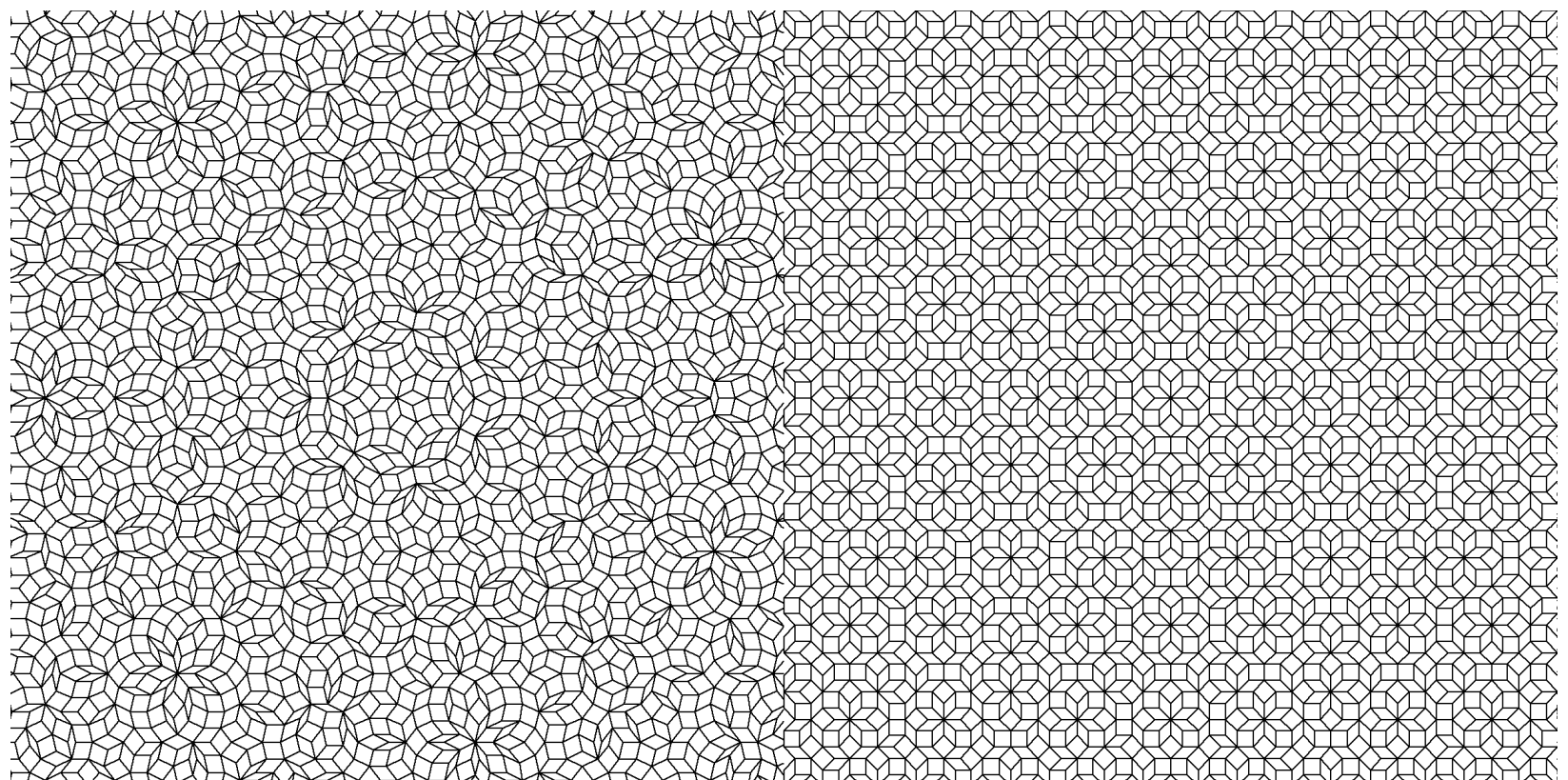

Figure 13. Arrangements of cells from canonical projection from 7-d and 8-d.

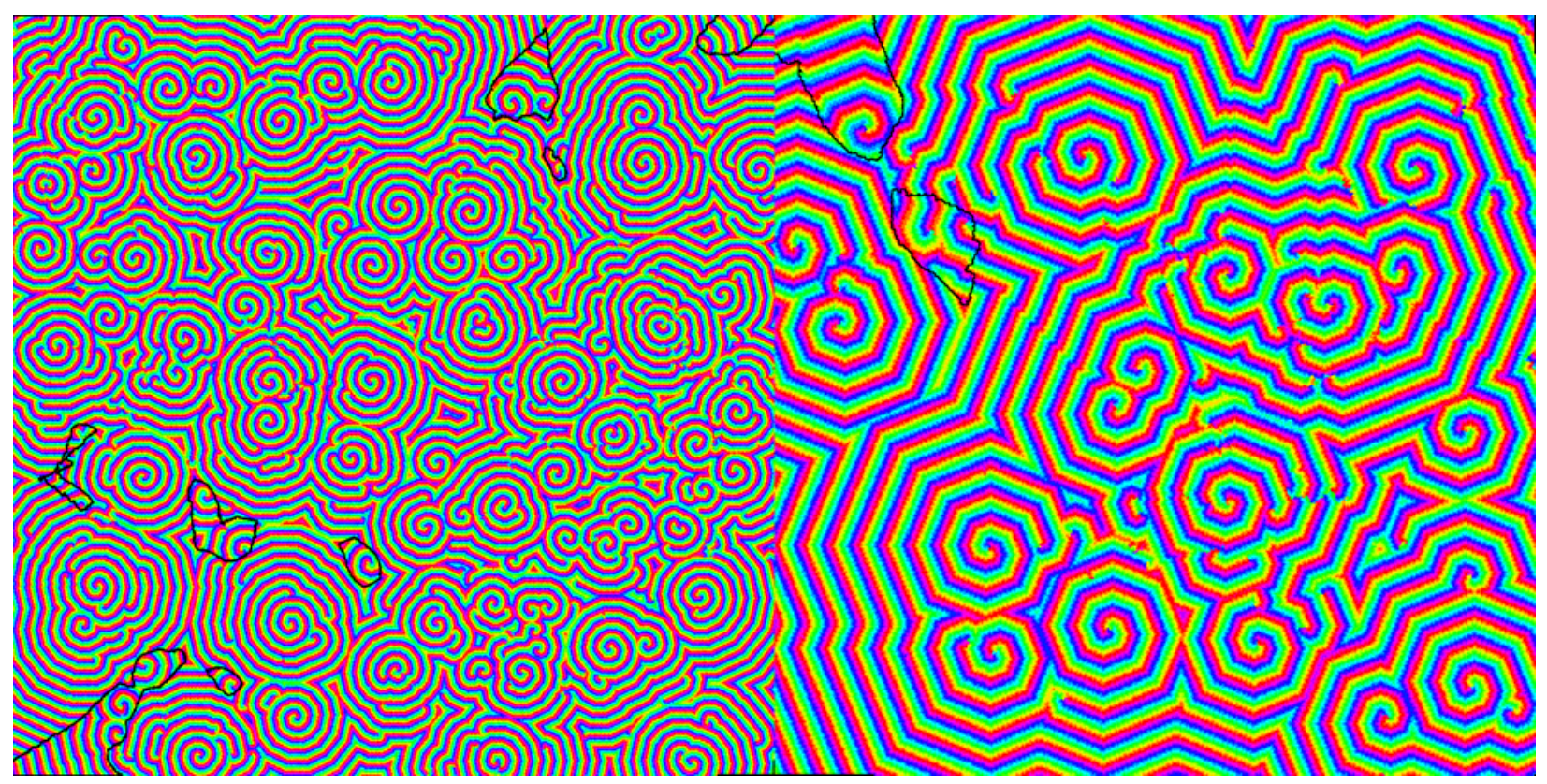

Figure 14. Spiral formation on a quasi-crystals from canonical projection from 7-d and 8-d. 